\title{
Polarization in relativistic heavy ion collisions: a theoretical per- spective
}

\author{
Francesco Becattini ${ }^{1 \star}$ \\ ${ }^{1}$ Dipartimento di Fisica e Astronomia, University of Florence, Via G. Sansone 1, I-50019, Sesto Fiorentino \\ (Firenze), Italy
}

\begin{abstract}
We review the theoretical framework for the calculation of particle polarization in relativistic heavy ion collisions within the hydrodynamical model. The covariant decomposition of the mean spin vector is presented and open theoretical issues addressed.
\end{abstract}

\section{Introduction}

Global polarization of $\Lambda$ hyperons in semi-peripheral relativistic heavy-ion collisions has been recently observed by the STAR experiment over a center-of-mass energy range between 7.7 and 200 $\mathrm{GeV}$ [1]. This finding confirms quantitative predictions based on local thermodynamic equilibrium of spin degrees of freedom [2] which provides a relation between polarization and relativistic vorticity, and it agrees quantitatively with the hydrodynamic model calculations to a very good degree of accuracy. In the hydrodynamic framework, the distinctive feature of polarization is its proportionality to the gradients of the combined temperature and velocity fields, so that its measurement is a stringent test of the hydrodynamic picture which is distinct and complementary to momentum spectra. Moreover, hydrodynamics and local thermodynamic equilibrium predict that polarization must be the same for particles and antiparticles, that is a C-even effect, in agreement with the observations. Conversely, if electromagnetic or other $\mathrm{C}$-odd fields were responsible for this phenomenon, polarization would be opposite for particles and antiparticles.

The experimental efforts, thus far, focussed on the search of the average global polarization of $\Lambda$ hyperons along the direction of the angular momentum of the plasma. This measurement requires the identification of the reaction plane in peripheral collision as well as its orientation, that is the direction of the total angular momentum vector $\mathbf{J}$. The average global polarization along $\mathbf{J}$ is also found to decrease rapidly as a function of center-of-mass energy [1], from few percent at $\sqrt{s_{\mathrm{NN}}}=O(10) \mathrm{GeV}$ to few permille at $\sqrt{s_{\mathrm{NN}}}=O(100) \mathrm{GeV}$. In the TeV energy range, at the LHC, the global polarization along $\mathbf{J}$ is not seen [3] as it is most likely beyond experimental sensitivity.

\section{The theoretical approaches to global polarization: a summary}

Particles produced in relativistic heavy ion collisions are expected to be polarized in peripheral collisions because of angular momentum conservation. At finite impact parameter, the Quark Gluon

\footnotetext{
^e-mail: becattini@fi.infn.it
} 
Plasma (QGP) has a finite angular momentum perpendicular to the reaction plane and some fraction thereof may be converted into spin of final state hadrons. Therefore, measured particles may show a finite mean global polarization along the angular momentum direction.

Early estimates of this effect [4] were based on the general idea that polarized quarks in the QGP stage of the production process would eventually give rise to polarized hadrons, making it possible to predict qualitative features of the final hadrons' polarization. It was then proposed $[5,6]$ that polarization can be calculated assuming that the spin degrees of freedom are at local thermodynamical equilibrium at the hadronization stage in much the same way as the momentum degrees of freedom. In other words, polarization can be predicted by extending the familiar Cooper-Frye formula to particles with spin. A specific derivation was presented in refs. [2, 5] where it was pointed out that the hydrodynamical quantity steering the polarization is the thermal vorticity, that is (minus) the antisymmetric part of the gradient of the four-temperature field $\beta=(1 / T) u$ where $T$ is the proper temperature and $u$ the hydrodynamic four-velocity:

$$
\varpi_{\mu v}=-\frac{1}{2}\left(\partial_{\mu} \beta_{v}-\partial_{v} \beta_{\mu}\right)
$$

Particularly, the first-order expansion of the polarization in terms of thermal vorticity was obtained in ref. [2] for hadrons with spin 1/2 (lately recovered with a different method in ref. [7]), yet its extension to higher spins could be derived from the corresponding global equilibrium expression [8].

This theoretical work made it possible to make definite quantitative predictions of global $\Lambda$ polarization in nuclear collisions from hydrodynamic calculations, with a resulting mean value ranging from some permille to some percent [9-11], with an apparently strong dependence on the initial conditions, particularly on the initial longitudinal velocity field. Calculations of vorticity in relativistic heavy ion collisions - which could be then turned into a polarization map - were also recently presented in ref. [12]. To complete the theoretical overview on the subject, it should be pointed out that different approaches, as well as additional mechanisms, to the $\Lambda$ polarization in relativistic nuclear collisions were proposed in refs. [13-16].

\section{Notation}

In this paper we use the natural units, with $\hbar=c=K=1$.

The Minkowskian metric tensor is $\operatorname{diag}(1,-1,-1,-1)$; for the Levi-Civita symbol we use the convention $\epsilon^{0123}=1$.

Operators in Hilbert space will be denoted by a large upper hat, e.g. $\widehat{T}$ while unit vectors with a small upper hat, e.g. $\hat{v}$.

\section{Hydrodynamics and the local equiibrium density operator}

The proper relativistic extension of the spin concept, for massive particles, requires the introduction of a spin four-vector operator. This is defined as follows:

$$
\widehat{S}^{\mu}=-\frac{1}{2 m} \epsilon^{\mu \nu \rho \lambda} \widehat{J}_{v \rho} \widehat{p}_{\lambda}
$$

where $\widehat{J}$ and $\widehat{p}$ are the angular momentum operator and four-momentum operator of a single particle. As it can be easily shown, the spin four-vector operator commutes with the four-momentum operator (hence it is a compatible observable) and it is space-like on free particle states as it is orthogonal to the four-momentum:

$$
\widehat{S}^{\mu} \widehat{p}_{\mu}=0
$$


and has thus only three independent components.

The mean spin $S^{\mu}$ and polarization $P^{\mu}$ four-vectors can now be defined as the mean values with the suitable density operators, that is:

$$
S^{\mu}=\left\langle\widehat{S}^{\mu}\right\rangle \equiv \operatorname{tr}\left(\widehat{\rho} \widehat{S}^{\mu}\right)
$$

and

$$
P^{\mu}=\left\langle\widehat{S}^{\mu}\right\rangle / S
$$

The crucial role in the calculation of (4) for the fluid produced in relativistic heavy ion collisions is played by the density operator. For a system at local thermodynamic equilibrium, this reads [17]:

$$
\widehat{\rho}_{\mathrm{LE}}=(1 / Z) \exp \left[-\int_{\Sigma} \mathrm{d} \Sigma_{\mu}\left(\widehat{T}^{\mu v} \beta_{v}-\widehat{\zeta j^{\mu}}\right)\right]
$$

where $\beta$ is the four-temperature vector, $\widehat{T}$ the stress-energy tensor, $\widehat{j}$ a conserved current - like the baryon number - and $\zeta=\mu / T$.

If we wish to calculate the mean value of a local operator $\widehat{O}(x)$ (such as, for instance the stressenergy tensor $\widehat{T}$, or the current $\widehat{j}$ )

$$
O(x)=\operatorname{tr}(\widehat{\rho} \widehat{O}(x))
$$

and if the fields $\beta, \zeta$ vary significantly over a distance which is much larger than the typical microscopic length (indeed the hydrodynamic limit), then they can be Taylor expanded in the density operator starting from the point $x$ where the mean value $O(x)$ is to be calculated. The leading terms in the exponent of (6) then become [17]:

$\widehat{\rho}_{\mathrm{LE}} \simeq \frac{1}{Z_{\mathrm{LE}}} \exp \left[-\beta_{v}(x) \widehat{P}^{v}+\xi(x) \widehat{Q}-\frac{1}{4}\left(\partial_{v} \beta_{\lambda}(x)-\partial_{\lambda} \beta_{v}(x)\right) \widehat{J}_{x}^{\lambda v}+\frac{1}{2}\left(\partial_{v} \beta_{\lambda}(x)+\partial_{\lambda} \beta_{v}(x)\right) \widehat{L}_{x}^{\lambda v}+\nabla_{\lambda} \xi(x) \widehat{d}_{x}^{\lambda}\right]$.

where the last two terms with the shear tensor and the gradient of $\zeta$ are dissipative and vanish at equilibrium. The $\nabla_{\lambda}$ operator stands for:

$$
\nabla_{\lambda}=\partial_{\lambda}-u_{\lambda} u \cdot \partial
$$

as usual in relativistic hydrodynamics. The term which is responsible for polarization is the one involving the generators of the Lorentz group $\widehat{J}_{x}$.

The polarization of particles in a fluid was then obtained by means of the spin tensor $\widehat{\mathcal{S}}$ which is the local operator associated to spin density along with an ansatz about the form of the covariant Wigner function [2]. The mean spin vector of $1 / 2$ particles with four-momentum $p$, produced around point $x$ at the leading order in the thermal vorticity is then found to be:

$$
S^{\mu}(x, p)=-\frac{1}{8 m}\left(1-n_{F}\right) \epsilon^{\mu \rho \sigma \tau} p_{\tau} \varpi_{\rho \sigma}
$$

where $n_{F}=(1+\exp [\beta(x) \cdot p-v(x) Q / T(x)]+1)^{-1}$ is the Fermi-Dirac distribution and $\varpi(x)$ is given by eq. 1 . The eq. (9) has been recovered with a different approach in ref. [7]. This formula is suitable for the situation of relativistic heavy ion collisions, where one deals with a local thermodynamic equilibrium hypersurface $\Sigma$ where hydrodynamic stage ceases and particle description sets in. 


\section{Covariant decomposition of the spin vector in a relativistic fluid}

To gain insight into the physics of polarization in a relativistic fluid, it is very useful to decompose the gradients of the four-temperature vector in the eq. (9). We start off with the seperation of the gradients of the comoving temperature and four-velocity field:

$$
\partial_{\mu} \beta_{v}=\partial_{\mu}\left(\frac{1}{T}\right)+\frac{1}{T} \partial_{\mu} u_{v}
$$

Then, we can introduce the acceleration and the vorticity vector $\omega^{\mu}$ with the usual definitions:

$$
\begin{aligned}
A^{\mu} & =u \cdot \partial u^{\mu} \\
\omega^{\mu} & =\frac{1}{2} \epsilon^{\mu \nu \rho \sigma} \partial_{\nu} u_{\rho} u_{\sigma}
\end{aligned}
$$

The antisymmetric part of the tensor $\partial_{\mu} u_{v}$ can then be expressed as a function of $A$ and $\omega$ :

$$
\frac{1}{2}\left(\partial_{\nu} u_{\mu}-\partial_{\mu} u_{v}\right)=\frac{1}{2}\left(A_{\mu} u_{v}-A_{\nu} u_{\mu}\right)+\epsilon_{\mu \nu \rho \sigma} \omega^{\rho} u^{\sigma}
$$

therafter plugged into the (9) to give:

$$
\begin{aligned}
S^{\mu}(x, p) & =\frac{1}{8 m}\left(1-n_{F}\right) \epsilon^{\mu \nu \rho \sigma} p_{\sigma} \nabla_{v}(1 / T) u_{\rho} \\
& +\frac{1}{8 m}\left(1-n_{F}\right) 2 \frac{\omega^{\mu} u \cdot p-u^{\mu} \omega \cdot p}{T} \\
& -\frac{1}{8 m}\left(1-n_{F}\right) \frac{1}{T} \epsilon^{\mu \nu \rho \sigma} p_{\sigma} A_{v} u_{\rho}
\end{aligned}
$$

Hence, polarization stems from three contributions: a term proportional to the gradient of temperature, a term proportional to the vorticity $\omega$, and a term proportional to the acceleration. Further insight into the nature of these terms can be gained by choosing the particle rest frame, where $p=(m, \mathbf{0})$ and restoring the natural units. The eq. (10) then certifies that the spin in the rest frame is proportional to the following combination:

$$
\mathbf{S}^{*}(x, p) \propto \frac{\hbar}{K T^{2}} \gamma \mathbf{v} \times \nabla T+\frac{\hbar}{K T} \gamma\left(\omega-(\omega \cdot \mathbf{v}) \mathbf{v} / c^{2}\right)+\frac{\hbar}{K T} \gamma \mathbf{A} \times \mathbf{v} / c^{2}
$$

where $\gamma=1 / \sqrt{1-v^{2} / c^{2}}$ and all three-vectors, including vorticity, acceleration and velocity, are observed in the particle rest frame.

The three independent contributions are now well discernible in eq. (13). The second term scales like $\hbar \omega / K T$ and is the one already known from non-relativistic physics, proportional to the vorticity vector seen by the particle in its motion amid the fluid, with an additional term vanishing in the nonrelativistic limit. The third term is a purely relativistic one and scales like $\hbar A / K T c^{2}$; it is usually overwhelmingly suppressed, except in heavy ion collisions where the acceleration of the plasma is huge ( $A \sim 10^{30} \mathrm{~g}$ at the outset of hydrodynamical stage). The first term, instead, is a new nonrelativistic term [2] and applies to situations where the velocity field is not parallel to the temperature gradient. For ideal uncharged (thus relativistic) fluids, this term is related to the acceleration term because the equations of motion reduce to:

$$
\nabla_{\mu} T=T A_{\mu} / c^{2}
$$

Therefore, being the QGP a quasi-ideal fluid and almost uncharged at very high energy, the first and third term are tightly related. It can be shown that they contribute non-trivially to the final predicted polarization [18]. 


\section{Open theoretical issues}

Specifying the mean spin vector at some point $x$ requires the spin tensor $\widehat{\mathcal{S}}$, what was used in ref. [2] to obtain the function in eq. (9). However, in relativistic heavy ion collisions, we do not measure spin at some spacetime point, but only as a function of particle momentum. In formulae, what we can do is to measure the mean spin after an integration of (9) over the freeze-out or particlization hypersurface $\Sigma$.

$$
S^{\mu}(p)=\frac{\int d \Sigma_{\lambda} p^{\lambda} f(x, p) S^{\mu}(x, p)}{\int d \Sigma_{\lambda} p^{\lambda} f(x, p)}
$$

It is presently an unsettled issue whether a formula like (14) can be obtained without using the spin tensor, that is in a quantum field theory where the stress-energy tensor is the symmetrized Belinfante tensor. The issue is a relevant one for if (14) was dependent on a spin tensor, $\widehat{\mathcal{S}}$ would acquire a full physical meaning and relativistic hydrodynamics - to say the least - should be extended [19] to fluids with spin.

Another major theoretical problem is to find an exact solution for the polarization at global thermodynamic equilibrium with $\varpi=$ const $\neq 0$, that is when the (6) becomes:

$$
\rho=\frac{1}{Z} \exp \left[-b_{\mu} \widehat{P}^{\mu}+\frac{1}{2} \varpi_{\mu \nu} \widehat{J}^{\mu \nu}+\zeta \widehat{Q}\right]
$$

Indeed, the formulae (9),(14) are first-order expansions in thermal vorticity and based on an educated ansatz on the Wigner function of the Dirac field with the density operator (15). Even though the formula (9) appears to be the only reasonable first-order expression, finding the exact solution would certainly be a benchmark in the theory of relativistic fluids with polarization and a crucial tool to settle open issues.

\section{Acknowledgments}

I am greatly indebted with I. Karpenko for his invaluable help in preparing this talk. The author would like to thank the organizers for their excellent work in arranging a very enjoyable conference and for their patience in waiting for my contribution to the proceedings.

\section{References}

[1] L. Adamczyk et al. [STAR Collaboration], Nature 548, 62 (2017)

[2] F. Becattini, V. Chandra, L. Del Zanna and E. Grossi, Annals Phys. 338, 32 (2013).

[3] M. Konyushikhin for the ALICE Coll., Global polarization of $\Lambda$ hyperons in $\mathrm{Pb}-\mathrm{Pb}$ collisions at $2.76 \mathrm{TeV}$, poster presented at the conference Quark Matter 2017, Chicago, USA.

[4] Z. T. Liang and X. N. Wang, Phys. Rev. Lett. 94, 102301 (2005); erratum ibidem 96, 039901(E) (2006).

[5] F. Becattini and F. Piccinini, Annals Phys. 323, 2452 (2008).

[6] F. Becattini, F. Piccinini and J. Rizzo, Phys. Rev. C 77, 024906 (2008).

[7] R. h. Fang, L. g. Pang, Q. Wang and X. n. Wang, Phys. Rev. C 94, no. 2, 024904 (2016).

[8] F. Becattini, I. Karpenko, M. Lisa, I. Upsal and S. Voloshin, Phys. Rev. C 95, no. 5, 054902 (2017).

[9] F. Becattini, L. Csernai and D. J. Wang, Phys. Rev. C 88, no. 3, 034905 (2013); Erratum: [Phys. Rev. C 93, no. 6, 069901 (2016)] 
[10] F. Becattini et al., Eur. Phys. J. C 75, no. 9, 406 (2015).

[11] L. G. Pang, H. Petersen, Q. Wang and X. N. Wang, Phys. Rev. Lett. 117, no. 19, 192301 (2016).

[12] Y. Jiang, Z. W. Lin and J. Liao, Phys. Rev. C 94, no. 4, 044910 (2016) Erratum: [Phys. Rev. C 95, no. 4, 049904 (2017)].

[13] A. Ayala, E. Cuautle, G. Herrera and L. M. Montano, Phys. Rev. C 65, 024902 (2002).

[14] C. d. C. Barros, Jr. and Y. Hama, Phys. Lett. B 699, 74 (2011).

[15] M. I. Baznat, K. K. Gudima, A. S. Sorin and O. V. Teryaev, Phys. Rev. C 93031902 (2016).

[16] A. Aristova, D. Frenklakh, A. Gorsky and D. Kharzeev, arXiv:1606.05882 [hep-ph].

[17] F. Becattini, L. Bucciantini, E. Grossi and L. Tinti, Eur. Phys. J. C 75, no. 5, 191 (2015).

[18] I. Karpenko, talk given at this conference; I. Karpenko and F. Becattini, in preparation.

[19] W. Florkowski, B. Friman, A. Jaiswal and E. Speranza, arXiv:1708.04035 [hep-ph]. 